\title{
UNA ESPECIE NUEVA DE LELLINGERIA (FILICALES: POLYPODIACEAE) PARA COSTA RICA, PANAMÁ Y COLOMBIA
}

\author{
Alexander Fco. Rojas Alvarado \\ Jardín Botánico Lankester. Universidad de Costa Rica. \\ Apdo.1031-7050, Cartago, Costa Rica. alfrojasa@yahoo.com
}

\begin{abstract}
In this paper a new species of Lellingeria is described: L. brenesii. This species is characterized by small size, long segment with crenate margin and superficial sori, and is known from Costa Rica, Panama and Colombia.
\end{abstract}

RESUMEN: En este trabajo una especie nueva de Lellingeria se describe: L. brenesii. Esta especie se caracteriza por ser pequeña, los segmentos largos con margen crenado y el soro superficial, se encuentra en Costa Rica, Panamá y Colombia.

Key Words: Lellingeria, new species, Polypodiaceae, Colombia, Costa Rica, Panama.

El género Lellingeria fue descrito por Smith, Moran \& Bishop (1991) y diferenciado de otros géneros de Polypodiaceae (grupo Grammitidae) por tener el rizoma radialmente simétrico, los pelos desigualmente furcados y las escamas del rizoma clatradas. También muchas de las especies del género presentan escamas del rizoma con el margen setoso y el soro foveado; caracteres que fueron confirmados por Moran \& Smith (1995).

En las últimas tres décadas son pocas las especies nuevas descritas y las que han sido combinadas, entre ellas: Grammitis barbensis Lellinger [=L. barbensis (Lellinger) A.R. Sm. \& R.C. Moran], G. micula Lellinger $[=$ L. melanotrichia (Baker) A.R. Sm. \& R.C. Moran] y G. pseudomichellae Lellinger [=L. pseudomichellae (Lellinger) A.R. Sm. \& R.C. Moran] (Lellinger 1985); G. hellwigii Mickel \& Beitel $[=$ L. hellwigii (Mickel \& Beitel) A.R. Sm. \& R.C. Moran], y G. prionodes Mickel \& Beitel $[=L$. prionodes (Mickel et Beitel) A.R. Sm. \& R.C. Moran] (Mickel \& Beitel 1988); G. sinuosa A.R. Sm. [=L. sinuosa (A.R. Sm.) A.R. Sm. \& R.C. Moran] (Smith 1990); con las correspondientes combinaciones (Smith Moran \& Bishop 1991); y $L$. cilolepis (C. Chr.) A.R. Sm., L. dissimulans (Maxon) A.R. Sm. y L. hombersleyi (Maxon) A.R. Sm. (Smith 1995); L. brasiliensis (Rosenst.) Labiak y L. pumila Labiak (Labiak 2000). En este artículo una especie nueva más es descrita.

\section{Lellingeria brenesii A. Rojas, sp. nov.}

TIPO: Costa Rica. Alajuela: Cantón de San Ramón, Reserva Biológica Alberto Manuel Brenes, Sendero Terciopelo, $10^{\circ} 12^{\prime} 45^{\prime \prime} \mathrm{N}, 84^{\circ} 36^{\prime} 25^{\prime \prime} \mathrm{W}, 1000-$ 1200 m, 2 jul 2006, A. Rojas \& E. Serrano 7193 (holotype: CR; isotypes: MO, NY, USJ). FIG. 1.

A Lellingeria suprasculpta (H. Christ) A.R. Sm. et R.C. Moran frondibus brevioribus, segmentis angustioribus et crenatis (adversus pinnatifida) et soro superficiali (adversus foveatum) differt.

Rizoma $1 \mathrm{~mm}$ de diámetro aproximadamente, compacto, con escamas $1.0-1.5 \times$ ca. $0.5 \mathrm{~mm}$, ovadas a lanceoladas, pardo-grisáceas, concolores, clatradas, margen y superficie ciliados; frondas 2-12 (-20) cm de largo; estípite ausente o menor de $0.5 \mathrm{~cm}$ de largo, con pelos 0.1-0.3 mm de largo, hialinos a amarillentos, varias veces desigualmente furcados; lámina de 0.7-1.7 cm de ancho, pinnatisecta a 1-pinnada, linear-elíptica, base cuneada a atenuada, ápice agudo; raquis recto (no flexuoso), pardo-grisáceo a negro, medianamente pubescente en ambas superficies (a veces el haz glabrescente), los tricomas 0.1-0.3 mm de largo, hialinos a amarillentos, varias veces desigualmente furcados, margen peloso, estos de 0.1-0.3 mm de largo, simples, hialinos, dispersos a medianamente densos (a veces solo presentes en las incisiones); pinnas de 3-8 x 1-3 $\mathrm{mm}$, lanceoladas a linear-lanceoladas, base sésil, ápice agudo a acuminado, onduladas a crenadas, inclinadas 


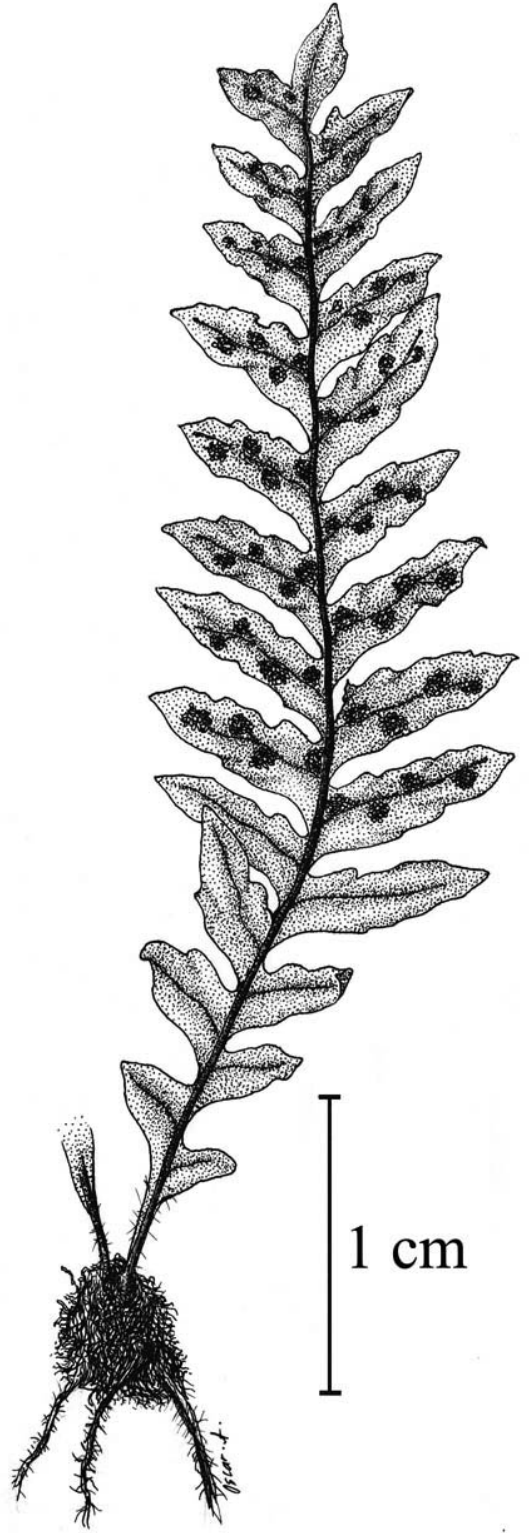

FIgura 1. Lellingeria brenesii (A. Rojas \& E. Serrano 7193, CR). a - Hábito.

45-75 con respecto a la costa, con 1-3 pares de lóbulos por pinna, a veces el lóbulo basal acroscópico más desarrollado; costas rectas, de color pajizo (a veces negruzcas en la base), pelosas a glabrescentes en el envés, los pelos como en el raquis; hidatodos visibles, 4-7 por pinna fértil, negros, resaltados en las pinnas fértiles; nervaduras fértiles no visibles; soros redondeados, superficiales; cápsulas esporangiales glabras.
Distribución: Se encuentra en la cordillera de Tilarán en Costa Rica, serranías del Darién en Panamá y Alto del Buey en Colombia a 800-1400 m.

PARATipos: COSTA RICA. Alajuela: San Ramón, Reserva Biológica Alberto Manuel Brenes, sendero a Bajo Jamaical, fila antes de bajar al río Jamaical, $10^{\circ} 14^{\prime} 15^{\prime \prime} \mathrm{N}, 84^{\circ} 35^{\prime} 50^{\prime \prime} \mathrm{W}, 800-1000 \mathrm{~m}, 5$ sept 2006, A. Rojas \& J. Cervantes 7352 (CR, F, INB, K, MO, NY, SEL, US, USJ). PANAMA. Darién: ridge top areas $\mathrm{N}$ of cerro Pirre, between cerro Pirre top and rancho Plástico, 1200-1400 m, 14 nov 1977, J. Folsom et al. 6313 (CR). COLOMBIA. Chocó: NW side of Alto del Buey trail, along ridge from de confluence of the fork of the río Mutatá above ten río Dos Bocas to the top of Alto del Buey, 7 febr 1971, D. Lellinger \& E. de la Sota 306 (CR).

Etimología: esta especie es dedicada a Alberto Manuel Brenes, quien hizo importantes aportes al conocimiento botánico de la cordillera de Tilarán, en particular en las cercanías de San Ramón.

La nueva especie se parece a Lellingeria suprasculpta (H. Christ) A.R. Sm. \& R.C. Moran por las pinnas incisas y por un lóbulo acroscópico más desarrollado, pero difiere por las escamas del rizoma setosas (vs. glabras), lámina crenada (vs. pinnatifida), raquis pubescente (vs. glabro), pinnas más pequeñas (3-8 $\times 1$ $3 \mathrm{~mm}$ vs. $10-25 \times 3-4 \mathrm{~mm}$ ) y por los soros superficiales (vs. foveados). En Mesoamérica es similar a L. melanotrichia (Baker) A. R. Sm. \& R. C. Moran por las escamas del rizoma setulosas y las pinnas incisas, pero difiere por características similares a $L$. suprasculpta y por los pelos del raquis que son varias veces desigualmente furcados (vs. simples). También es similar a $L$. sinuosa (A.R. Sm.) A.R. Sm. \& R.C. Moran por el raquis negro y por la forma e incisión de las pinnas, pero se le reconoce por las escamas del rizoma con margen ciliado (vs. entero), estípite y raquis densamente cubierto de pelos desigualmente furcados (vs. estípite glabro y raquis glabrescente con pelos simples), pinnas más cortas (3-8 mm de largo vs. 7-12 mm) y con el margen setuloso (vs. glabro) y costas setulosas (vs. glabras). Probablemente es más parecida a $L$. randallii (Maxon) A.R. Sm. \& R.C. Moran por los segmentos lobulados y los soros superficiales, pero difiere por tener el raquis recto (vs. flexuoso) y los segmentos lanceolados a linear lanceolados (vs. ovados) (Figs. 1 y 2). 


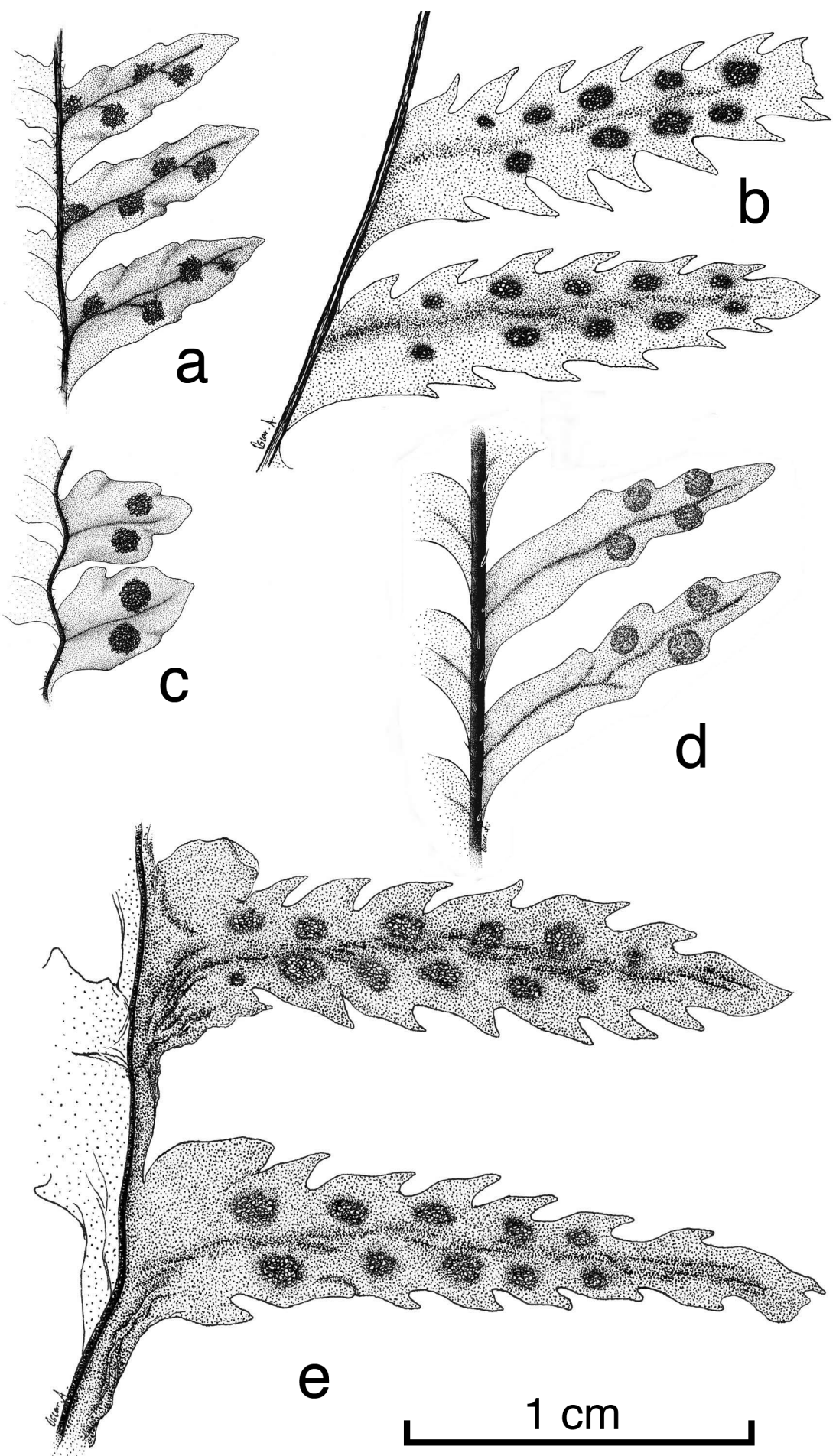

Figura 2. Comparación de las pinnas de: a - Lellingeria brenesii (A. Rojas \& E. Serrano 7193, CR). b - L. melanotrichia (A. Rojas \& A. Soto 4251, CR). c - L. randallii (H. Werff \& C. Hardeveld 6949, MO). d - L. sinuosa (R. Maguire 24556, NY). e - L. suprasculpta (A. Rojas et al. 4071, CR). 
Agradecimientos. A Cyril Nelson por la traducción de la diagnosis al latín, a Oscar Venegas por las excelentes ilustraciones, a Hamer Salazar por ayudarme en la logística para trabajar con el inventario de la Reserva Biológica Alberto M. Brenes, al curador del herbario del Museo Nacional de Costa Rica (CR) Armando Ruiz, por permitirme utilizar las colecciones. A los revisores anónimos por sus comentarios que apoyaron la hipótesis de este estudio.

\section{LITERATURA CITADA}

Labiak, P.H. 2000. New species and new combinations in neotropical Grammitidaceae (Pteridophyta). Brittonia 52: 246-255.

Lellinger, D.B. 1985. Nomenclatural and taxonomic notes on the Pteridophytes of Costa Rica, Panama and Colombia, II. Proc. Biol. Soc. Wash. 98: 366-390.
Mickel, J.T. \& J.M. Beitel. 1988. Pteridophyte Flora of Oaxaca, Mexico. Mem. New York Bot. Gard. 46: 193206.

Moran, R.C. \& A.R. Smith. 1995. Lellingeria. In: R.C. Moran \& R. Riba (eds.). Flora Mesoamericana. Volumen 1. Psilotaceae a Salviniaceae. Univ. Nac. Autónoma de México. México, D. F., p. 376-380.

Rojas, A.R. 2006. Nuevos taxa en Grammitidaceae (Pteridophyta) de Costa Rica. Lankesteriana 6: 95-100.

Smith, A.R. 1990. Pteridophytes of the Venezuelan Guayana: new species. Ann. Missouri Bot. Gard. 77: 249-273.

Smith, A.R. 1995. New combinations in Neotropical Grammitidaceae (Pteridophyta). Novon: 20-21.

Smith, A.R., R.C. Moran \& L.E. Bishop. 1991. Lellingeria, a new genus of Grammitidaceae. Amer. Fern J. 81: 76-88. 